\title{
Expression of Pregnancy Up-regulated Non-ubiquitous Calmodulin Kinase (PNCK) in Hepatocellular Carcinoma
}

\author{
YOON AH CHO ${ }^{1,2}$, SANGJOON CHOI $^{1}$, SUJIN PARK $^{1}$, CHEOL-KEUN PARK $^{1,3}$ and SANG YUN HA ${ }^{1}$ \\ ${ }^{1}$ Department of Pathology and Translational Genomics, Samsung Medical Center, \\ Sungkyunkwan University School of Medicine, Seoul, Republic of Korea; \\ ${ }^{2}$ Department of Pathology, Hallym University Sacred Heart Hospital, \\ Hallym University College of Medicine, Anyang, Republic of Korea; \\ ${ }^{3}$ Anatomic Pathology Reference Lab, Seegene Medical Foundation, Seoul, Republic of Korea
}

\begin{abstract}
Background/Aim: Pregnancy up-regulated nonubiquitous calmodulin kinase (PNCK) is a member of calmodulin kinase, and overexpression of PNCK with involvement in carcinogenesis have been reported in HER-2 amplified breast cancer, clear cell renal cell carcinoma and nasopharygeal carcinoma. However, the expression of PNCK and its clinical implication have not been elucidated in hepatocellular carcinoma (HCC). Materials and Methods: We investigated PNCK expression at both the protein and mRNA level using immunohistochemistry (IHC) and microarray gene expression profiling in HCC tissue samples, and evaluated its association with clinicopathological parameters and their potential prognostic significance. Results: High PNCK protein expression and high PNCK mRNA level was observed in $61.7 \%$ and $34.7 \%$ of total HCC cases, respectively. PNCK mRNA level was higher in tumor tissues than in background non-tumor tissues, and significantly correlated with protein expression by IHC. High PNCK expression was associated with higher Edmondson grade, intrahepatic metastasis, microvascular invasion and higher AFP levels. Patients with high PNCK expression showed shorter recurrence-free survival and disease-specific survival, and high mRNA expression of PNCK was an independent prognostic factor in disease-specific survival.
\end{abstract}

This article is freely accessible online.

Correspondence to: Sang Yun Ha, MD, Ph.D., Department of Pathology and Translational Genomics, Samsung Medical Center, Sungkyunkwan University School of Medicine, 81 Irwon-ro, Gangnam-gu, Seoul 06351, Republic of Korea. Tel: +82 234101586, Fax: +82 234100025, e-mail: sangyun.ha@skku.edu

Key Words: Hepatocellular carcinoma, prognosis, PNCK, EGFR, calmodulin.
Conclusion: Up-regulation of PNCK expression as well as its association with poor prognosis was demonstrated in HCC. PNCK might be a prognostic biomarker of HCC, and could be a potential candidate therapeutic target.

Hepatocellular carcinoma (HCC) shows a high incidence of tumor recurrence and metastasis, causing a poor prognosis. Sorafenib has been recognized as the most effective treatment for advanced HCC and new targeted agents such as regorafenib and lenvatinib, or programmed cell death protein1 immune checkpoint inhibitors such as nivolumab and pembrolizumab have also been approved by the US Food and Drug Administration (FDA). However, the application of these therapies remains limited (1-4). Therefore, the investigation of new therapeutic targets and reliable biomarkers is necessary for more effective treatment of $\operatorname{HCC}(5,6)$.

Pregnancy up-regulated non-ubiquitous calmodulin kinase $(P N C K)$ is a calmodulin kinase which is located in Xq28 and is expressed during fetal development and acts in a tissuespecific manner (7). There have been a few previous studies regarding PNCK expression with its involvement carcinogenesis in human epidermal growth factor receptor-2 (HER-2) amplified breast cancer, clear cell renal cell carcinoma and nasopharygeal carcinoma (8-10). PNCK expression was increased in tumor tissues compared to non-tumor tissues, and has been associated with proliferation of tumor cells in HER-2 amplified breast cancer and nasopharyngeal carcinoma $(8,9)$. In addition, overexpression of PNCK was related with a poor prognosis as well as poor differentiation, large tumor size, and advanced $\mathrm{T}$ and $\mathrm{N}$ stage in clear cell renal cell carcinoma (10). However, the expression of PNCK and its clinical implications have not been elucidated in HCC.

In this study, we investigated PNCK expression at both protein and mRNA level in HCC tissue samples, and evaluated its association with clinicopathologic parameters and prognostic significance. 

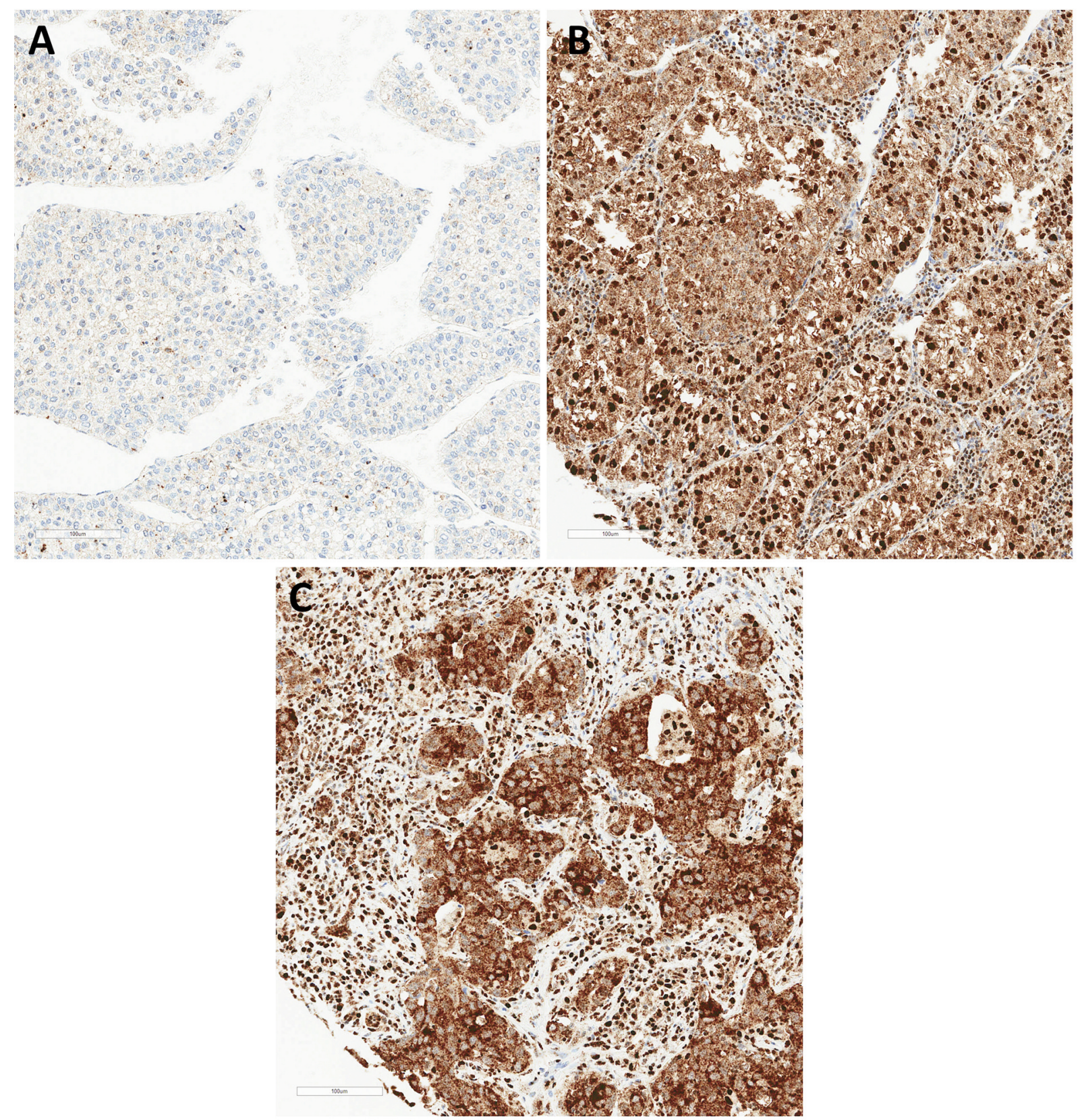

Figure 1. Representative figures of pregnancy up-regulated non-ubiquitous calmodulin kinase (PNCK) immunohistochemistry (IHC). (A) Tumor cells show negative or faint staining of PNCK IHC. (B-C) Tumor cells show nuclear or cytoplasmic staining of PNCK IHC.

\section{Materials and Methods}

Patients and specimens. Initially, a total of 291 patients with curative hepatectomy for primary HCC from July 2000 to May 2006 at the Samsung Medical Center, Seoul, Korea were enrolled. Eight patients with preoperative local treatment such as radiofrequency ablation, transarterial chemoembolization or radiotherapy and 17 patients with no sufficient tissue on tissue microarray (TMA) were excluded. Finally, 266 patients were included in the study.
Tumors with complete resection margins were confirmed by microscopic examination and no residual tumor one month after surgery was defined as curative resection. All tumor tissues were histologically confirmed. Tumor stages were assessed according to both the American Joint Committee on Cancer (AJCC) staging system, $8^{\text {th }}$ edition (11) and Barcelona Clinic Liver Cancer (BCLC) staging classification (12). Intrahepatic metastasis and multicentric occurrence were defined as in previously reported criteria (13). All patients were followed-up every 3 months after operation, with three 
A

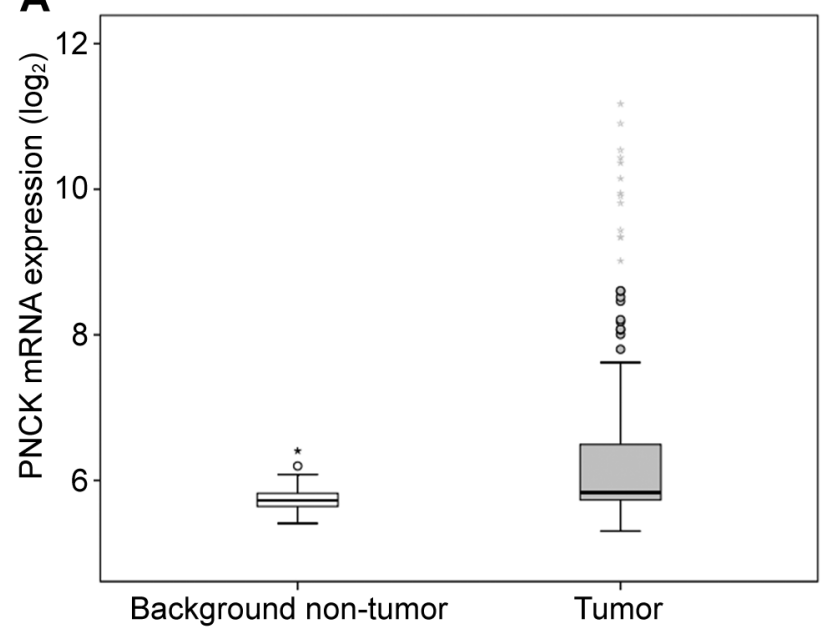

B

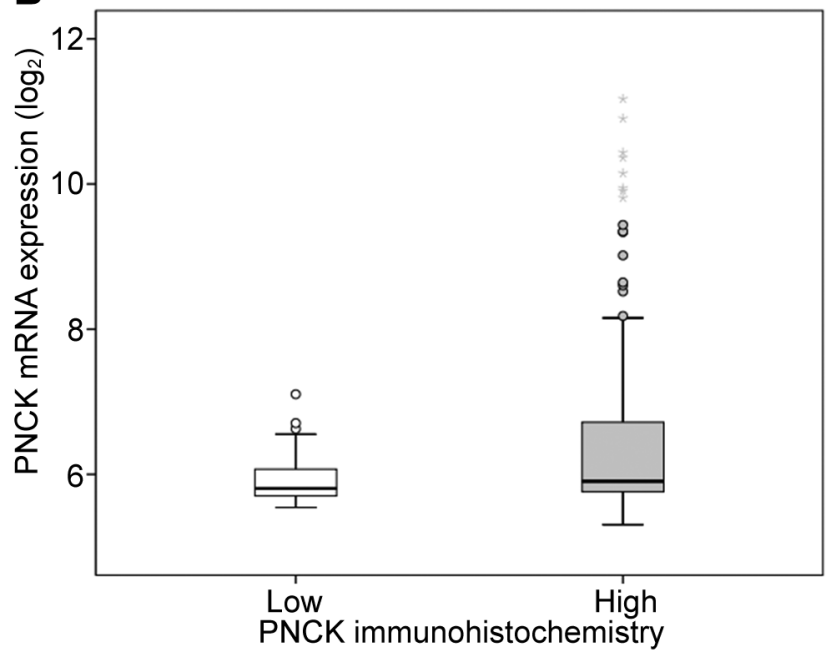

Figure 2. (A) mRNA expression level of pregnancy up-regulated non-ubiquitous calmodulin kinase (PNCK) in hepatocellular carcinoma tissues and background non-tumor liver tissues. (B) mRNA expression level of PNCK in low expression and high expression group by immunohistochemistry of PNCK.

phase dynamic computed tomography scans or magnetic resonance imaging and serum $\alpha$-fetoprotein (AFP) levels. Recurrence-free survival (RFS) or disease-specific survival (DSS) was defined as the difference between the date of surgery and the date of recurrence or HCC-related death, respectively, as previously described (14). Institutional Review Board of Samsung Medical Center approved this study and waived the informed consent.

Immunohistochemical studies. Immunohistochemistry was performed on tissue microarray consisting of two $2 \mathrm{~mm}$ cores of HCC tissue as previously described (15). The sections were incubated with a rabbit anti-PNCK antibody (HPA007458, 1:100, Sigma-Aldrich Inc., St. Louis, MO, USA) for 120 min by using the Ventana BenchMark XT Autostainer (Ventana Medical Systems Inc., Tucson, AZ, USA), after Heat Induced Epitope Retrieval (HIER) with CC1 for $92 \mathrm{~min}$. Antigen-antibody chromogenic reactions were developed for $12 \mathrm{~min}$ and detected using an OptiView DAB IHC Detection kit (Ventana Medical Systems Inc.). Normal brain tissue was used for positive control. No staining or weak intensity of IHC staining was considered as negative (Figure 1A), while moderate to strong intensity of nuclear or cytoplasmic IHC staining was considered as positive (Figure 1B and C). The proportion of positive PNCK IHC staining among the tumor was evaluated.

mRNA expression of PNCK. We used microarray gene expression profiling data that were obtained by using the same patient cohort (16). The data have been submitted in Gene Expression Omnibus (GSE 36376, http://www.ncbi.nlm.nih.gov/geo/). It consists of 240 HCC tissue and 193 adjacent non-tumor liver tissues. Among them, data from 219 HCC samples and 163 non-tumor liver samples were used for this study. The normalized values of PNCK (probe ID: ILMN_1697189) expression with base 2 logarithm were extracted.

Statistical analysis. X-tile bio-informatics software (Yale University, New Haven, CT, USA) (17) was used to determine the cut-off value of PNCK expression with the most significant difference in RFS.
Pearson's Chi square tests, Fisher's exact tests or Cochran Armitage test were used to analyze the relationships between PNCK expression and clinicopathologic parameters, as appropriate. The Mann-Whitney $U$-test was performed to compare PNCK mRNA expression between tumor and normal tissue. The Spearman test was used to evaluate correlation between IHC expression and mRNA expression of PNCK. The Kaplan-Meier method was used to analyze survival rates, and differences were compared using the log-rank test. Multivariate regression analysis was performed using a Cox proportional hazards model. Two-sided $p$-values $<0.05$ were considered statistically significant. Statistical analyses were conducted using IBM SPSS software for Windows (IBM Corp., Armonk, NY, USA).

\section{Results}

PNCK IHC staining in HCC in conjunction with clinicopathological features. PNCK IHC expression varied among HCC cases, which showed nuclear or cytoplasmic expression and heterogeneous staining (Figure 1). The proportion of positive staining in HCCs ranged from 0 to $100 \%$, with mean $27.19 \%$ and median $8.75 \%$. By using X-tile analysis (17), the best cut-off value for PNCK IHC expression associated with RFS was 5\%. High PNCK IHC expression was observed in $61.7 \%$ of HCCs, and was significantly related with age younger than 55 years-old $(p=0.007)$, higher Edmondson grade $(p=0.040)$, presence of microvascular invasion $(p=0.003)$, presence of intrahepatic metastasis $(p=0.027)$ and AFP level more than $200 \mathrm{ng} / \mathrm{ml}(p=0.002)$ (Table I).

PNCK $m$ RNA expression in HCCs. Mean value of normalized $P N C K$ mRNA expression in tumors was 6.39 (range=5.3011.17). The best cut-off value for $P N C K$ mRNA level associated with RFS was 6.09 , by X-tile analysis (17). PNCK 

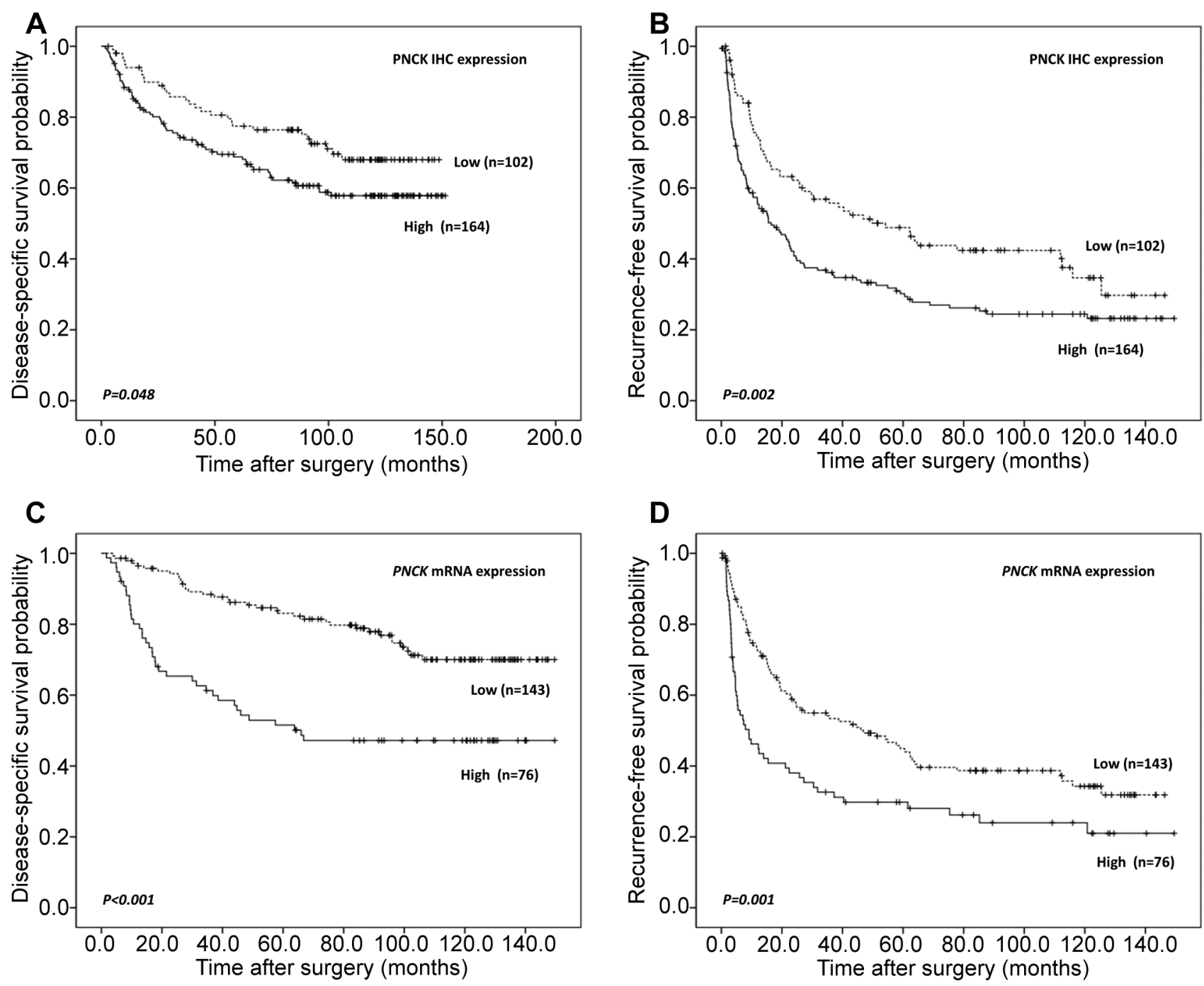

Figure 3. Kaplan-Meier survival curves according to pregnancy up-regulated non-ubiquitous calmodulin kinase (PNCK) expression; (A-B) PNCK protein expression (C-D) PNCK mRNA expression.

mRNA expression was significantly higher in tumor than in background liver tissues $(6.39 \pm 1.17$ vs. $5.74 \pm 0.14, p<0.001$, Figure 2A). PNCK mRNA expression was significantly correlated with PNCK IHC expression (Spearman's coefficient $=0.221, p=0.001$ ), and $P N C K$ mRNA expression was higher in high PNCK IHC expression group than in low group $(6.56 \pm 1.31$ vs $6.12 \pm 0.86, p=0.001$, Figure $2 \mathrm{~B})$.

High PNCK mRNA expression was observed in $34.7 \%$ of HCCs, and was significantly associated with larger tumor size $(p<0.001)$, higher Edmondson grade $(p=0.004)$, presence of microvascular invasion $(p<0.001)$, presence of major portal vein invasion $(p=0.009)$, presence of intrahepatic metastasis $(p<0.001)$, higher AJCC stage $(p<0.001)$, higher BCLC stage $(p<0.001)$ and AFP level more than $200 \mathrm{ng} / \mathrm{ml}$ $(p<0.001)$ (Table I).
Impact of PNCK expression on the survival of HCC patients. Patients with high expression of PNCK IHC showed shorter DSS ( $p=0.048$, Figure $3 \mathrm{~A})$ and RFS ( $p=0.002$, Figure 3B). Patients with high expression of PNCK mRNA showed shorter DSS $(p<0.001$, Figure 3C) and RFS ( $p=0.001$, Figure 3D). In multivariate analysis, $P N C K$ mRNA expression was an independent prognostic factor for DSS $[\mathrm{HR}=1.908(95 \%$ $\mathrm{CI}=1.183-3.078), p=0.008$ by the time-dependent Cox model], in addition to intrahepatic metastasis (Tables II and III).

\section{Discussion}

In this study, we elucidated that $P N C K$ expression at the mRNA level was up-regulated in HCC samples compared to background non-tumor liver tissue. High expression of 
Table I. The association between PNCK expression and clinicopathological parameters.

\begin{tabular}{|c|c|c|c|c|c|c|c|c|c|c|c|c|}
\hline \multirow[t]{2}{*}{ Category } & \multirow{2}{*}{$\begin{array}{l}\text { No. of } \\
\text { cases } \\
(\mathrm{n}=266)\end{array}$} & \multicolumn{4}{|c|}{ PNCK IHC expression } & \multirow[t]{2}{*}{$p$-Value } & \multirow{2}{*}{$\begin{array}{l}\text { No. of } \\
\text { cases } \\
(\mathrm{n}=219)\end{array}$} & \multicolumn{4}{|c|}{$P N C K$ mRNA level } & \multirow[t]{2}{*}{$p$-Value } \\
\hline & & \multicolumn{2}{|c|}{$\begin{array}{c}\text { High (\%) } \\
(\mathrm{n}=164)\end{array}$} & \multicolumn{2}{|c|}{$\begin{array}{c}\text { Low }(\%) \\
(\mathrm{n}=102)\end{array}$} & & & \multicolumn{2}{|c|}{$\begin{array}{c}\text { High (\%) } \\
(\mathrm{n}=76)\end{array}$} & \multicolumn{2}{|c|}{$\begin{array}{c}\text { Low }(\%) \\
(\mathrm{n}=143)\end{array}$} & \\
\hline & & \multicolumn{11}{|c|}{ Age (year) } \\
\hline$\leq 55$ & 158 & 108 & $(65.9)$ & 50 & $(49.0)$ & 0.007 & 130 & 50 & $(65.8)$ & 80 & $(55.9)$ & 0.194 \\
\hline$>55$ & 108 & 56 & $(34.1)$ & 52 & $(51.0)$ & & 89 & 26 & $(34.2)$ & 63 & $(44.1)$ & \\
\hline \multicolumn{13}{|l|}{ Gender } \\
\hline Male & 217 & 138 & $(84.1)$ & 79 & $(77.5)$ & 0.194 & 179 & 64 & $(84.2)$ & 115 & $(80.4)$ & 0.583 \\
\hline Female & 49 & 26 & $(15.9)$ & 23 & $(22.5)$ & & 40 & 12 & $(15.8)$ & 28 & (19.6) & \\
\hline \multicolumn{13}{|c|}{ Tumor size $(\mathrm{cm})$} \\
\hline$\leq 5.0$ & 178 & 109 & $(66.5)$ & 69 & $(67.6)$ & 0.894 & 148 & 33 & $(43.4)$ & 115 & $(80.4)$ & $<0.001$ \\
\hline$>5.0$ & 88 & 55 & $(33.5)$ & 33 & (32.4) & & 71 & 43 & $(56.6)$ & 28 & (19.6) & \\
\hline \multicolumn{13}{|c|}{ Edmondson grade } \\
\hline I & 29 & 15 & $(9.1)$ & 14 & (13.7) & 0.040 & 23 & 2 & $(2.6)$ & 21 & $(14.7)$ & 0.004 \\
\hline II & 216 & 131 & $(79.9)$ & 85 & $(83.3)$ & & 179 & 64 & $(84.2)$ & 115 & $(80.4)$ & \\
\hline III & 21 & 18 & $(11.0)$ & 3 & $(2.9)$ & & 17 & 10 & (13.2) & 7 & $(4.9)$ & \\
\hline \multicolumn{13}{|c|}{ Microvascular invasion } \\
\hline Absent & 118 & 61 & $(37.2)$ & 57 & $(55.9)$ & 0.003 & 97 & 12 & $(15.8)$ & 85 & $(59.4)$ & $<0.001$ \\
\hline Present & 148 & 103 & $(62.8)$ & 45 & $(44.1)$ & & 122 & 64 & $(84.2)$ & 58 & $(40.6)$ & \\
\hline \multicolumn{13}{|c|}{ Major portal vein invasion } \\
\hline Absent & 253 & 153 & $(93.3)$ & 100 & $(98.0)$ & 0.141 & 210 & 69 & $(90.8)$ & 141 & (98.6) & 0.009 \\
\hline Present & 13 & 11 & $(6.7)$ & 2 & $(2.0)$ & & 9 & 7 & $(9.2)$ & 2 & $(1.4)$ & \\
\hline \multicolumn{13}{|c|}{ Intrahepatic metastasis } \\
\hline Absent & 201 & 116 & $(70.7)$ & 85 & $(83.3)$ & 0.027 & 167 & 46 & $(60.5)$ & 121 & $(84.6)$ & $<0.001$ \\
\hline Present & 65 & 48 & $(29.3)$ & 17 & (16.7) & & 52 & 30 & $(39.5)$ & 22 & (15.4) & \\
\hline Multicentric oc & & & & & & & & & & & & \\
\hline Absent & 249 & 156 & $(95.1)$ & 93 & $(91.2)$ & 0.209 & 207 & 73 & $(96.1)$ & 134 & (93.7) & 0.755 \\
\hline Present & 17 & 8 & $(4.9)$ & 9 & $(8.8)$ & & 12 & 3 & $(3.9)$ & 9 & $(6.3)$ & \\
\hline AJCC T stage & & & & & & & & & & & & \\
\hline 1 & 42 & 25 & $(15.2)$ & 17 & (16.7) & 0.304 & 34 & 10 & $(13.2)$ & 24 & (16.8) & $<0.001$ \\
\hline 2 & 133 & 81 & (49.4) & 52 & $(51.0)$ & & 111 & 21 & $(27.6)$ & 90 & $(62.9)$ & \\
\hline 3 & 77 & 46 & $(28.0)$ & 31 & (30.4) & & 64 & 38 & $(50.0)$ & 26 & (18.2) & \\
\hline 4 & 14 & 12 & $(7.3)$ & 2 & $(2.0)$ & & 10 & 7 & $(9.2)$ & 3 & $(2.1)$ & \\
\hline BCLC stage & & & & & & & & & & & & \\
\hline $0-\mathrm{A}$ & 153 & 93 & $(56.7)$ & 60 & $(58.8)$ & 0.122 & 128 & 28 & $(36.8)$ & 100 & $(69.9)$ & $<0.001$ \\
\hline B & 98 & 58 & $(35.4)$ & 40 & (39.2) & & 81 & 40 & $(52.6)$ & 41 & (28.7) & \\
\hline $\mathrm{C}$ & 15 & 13 & $(7.9)$ & 2 & $(2.0)$ & & 10 & 8 & $(10.5)$ & 2 & $(1.4)$ & \\
\hline Albumin level & & & & & & & & & & & & \\
\hline$>3.5$ & 236 & 143 & $(87.2)$ & 93 & $(91.2)$ & 0.426 & 195 & 66 & $(86.8)$ & 129 & $(90.2)$ & 0.495 \\
\hline$\leq 3.5$ & 30 & 21 & (12.8) & 9 & $(8.8)$ & & 24 & 10 & $(13.2)$ & 14 & $(9.8)$ & \\
\hline AFP level (ng/ & & & & & & & & & & & & \\
\hline$\leq 200$ & 157 & 85 & $(53.5)$ & 72 & (73.5) & 0.002 & 131 & 29 & $(39.7)$ & 102 & (72.9) & $<0.001$ \\
\hline$>200$ & 100 & 74 & $(46.5)$ & 26 & (26.5) & & 82 & 44 & $(60.3)$ & 38 & $(27.1)$ & \\
\hline Etiology & & & & & & & & & & & & \\
\hline Non-viral & 32 & 20 & $(12.2)$ & 12 & (11.8) & 0.828 & 27 & 8 & $(10.5)$ & 19 & $(13.3)$ & 0.323 \\
\hline $\mathrm{HBV}$ & 206 & 129 & $(78.7)$ & 77 & $(75.5)$ & & 171 & 64 & $(84.2)$ & 107 & $(74.8)$ & \\
\hline $\mathrm{HCV}$ & 24 & 13 & $(7.9)$ & 11 & (10.8) & & 18 & 4 & $(5.3)$ & 14 & $(9.8)$ & \\
\hline HBV\&HCV & 4 & 2 & $(1.2)$ & 2 & $(2.0)$ & & 3 & 0 & $(0.0)$ & 3 & (2.1) & \\
\hline Non tumor live & & & & & & & & & & & & \\
\hline Cirrhosis & 133 & 85 & $(51.8)$ & 48 & $(47.1)$ & 0.528 & 106 & 36 & $(47.4)$ & 70 & $(49.0)$ & 0.887 \\
\hline Others & 133 & 79 & $(48.2)$ & 54 & (52.9) & & 113 & 40 & $(52.6)$ & 73 & $(51.0)$ & \\
\hline
\end{tabular}

Values are presented as number (\%). AJCC, American Joint Committee on Cancer; BCLC, Barcelona Clinic Liver Cancer; AFP, $\alpha$-fetoprotein; HBV, hepatitis B virus; $\mathrm{HCV}$, hepatitis $\mathrm{C}$ virus. ${ }^{\mathrm{a}} \mathrm{AFP}$ evaluation was not applicable in 9 cases. 
Table II. Univariate and multivariate analysis for disease-specific survival.

\begin{tabular}{|c|c|c|c|c|c|c|}
\hline \multirow[t]{3}{*}{ Category } & \multicolumn{6}{|c|}{ Disease specific survival } \\
\hline & \multicolumn{2}{|c|}{ Univariate } & \multicolumn{2}{|c|}{ Multivariate } & \multicolumn{2}{|c|}{ Multivariate } \\
\hline & $\operatorname{HR}(95 \% \mathrm{CI})$ & $p$-Value & HR $(95 \% \mathrm{CI})$ & $p$-Value & HR $(95 \%$ CI $)$ & $p$-Value \\
\hline \multicolumn{7}{|l|}{$\operatorname{Age}(y)^{*}$} \\
\hline$\leq 55$ & 1 & & & & & \\
\hline$>55$ & $0.972(0.641-1.474)$ & 0.895 & & & & \\
\hline \multicolumn{7}{|l|}{ Gender } \\
\hline Female & 1 & & & & & \\
\hline Male & $0.838(0.496-1.418)$ & 0.510 & & & & \\
\hline \multicolumn{7}{|c|}{ Tumor size $(\mathrm{cm})$} \\
\hline$\leq 5$ & 1 & & 1 & & 1 & \\
\hline$>5$ & $3.390(2.257-5.092)$ & $<0.001$ & $1.688(1.030-2.766)$ & 0.038 & $1.478(0.871-2.507)$ & 0.148 \\
\hline \multicolumn{7}{|c|}{ Microvascular invasion } \\
\hline Absent & 1 & & 1 & & 1 & \\
\hline Present & $3.536(2.206-5.667)$ & $<0.001$ & $1.249(0.675-2.309)$ & 0.479 & $1.281(0.645-2.544)$ & 0.479 \\
\hline \multicolumn{7}{|c|}{ Major portal vein invasion } \\
\hline Absent & 1 & & 1 & & 1 & \\
\hline Present & $5.628(2.900-10.922)$ & $<0.001$ & $1.248(0.608-2.561)$ & 0.545 & $1.481(0.657-3.336)$ & 0.343 \\
\hline \multicolumn{7}{|c|}{ Intrahepatic metastasis } \\
\hline Absent & 1 & & 1 & & 1 & \\
\hline Present & $6.431(4.257-9.715)$ & $<0.001$ & $4.492(2.556-7.896)$ & $<0.001$ & $5.410(3.318-8.822)$ & $<0.001$ \\
\hline \multicolumn{7}{|l|}{ Multicentricity } \\
\hline Absent & 1 & & & & & \\
\hline Present & $0.417(0.153-1.136)$ & 0.087 & & & & \\
\hline \multicolumn{7}{|c|}{ Edmonson grade } \\
\hline I-II & 1 & & 1 & & 1 & \\
\hline III & $2.345(1.249-4.402)$ & 0.008 & $1.207(0.620-2.347)$ & 0.580 & $1.020(0.470-2.215)$ & 0.959 \\
\hline \multicolumn{7}{|l|}{ AJCC T stage } \\
\hline $\mathrm{T} 1$ & 1 & & & & & \\
\hline $\mathrm{T} 2$ to $\mathrm{T} 4$ & $4.659(1.892-11.475)$ & 0.001 & & & & \\
\hline \multicolumn{7}{|l|}{ BCLC stage } \\
\hline 0 to $\mathrm{A}$ & 1 & & & & & \\
\hline B to $\mathrm{C}$ & $3.506(2.278-5.397)$ & $<0.001$ & & & & \\
\hline \multicolumn{7}{|c|}{ Serum albumin level } \\
\hline$>3.5 \mathrm{~g} / \mathrm{dl}$ & 1 & & & & & \\
\hline$\leq 3.5 \mathrm{~g} / \mathrm{dl}$ & $1.510(0.892-2.554)$ & 0.125 & & & & \\
\hline \multicolumn{7}{|c|}{ Serum AFP level } \\
\hline$\leq 200 \mathrm{ng} / \mathrm{ml}$ & 1 & & 1 & & 1 & \\
\hline$>200 \mathrm{ng} / \mathrm{ml}$ & $1.800(1.193-2.716)$ & 0.005 & $0.932(0.586-1.480)$ & 0.764 & $0.759(0.440-1.308)$ & 0.321 \\
\hline \multicolumn{7}{|l|}{ Etiology } \\
\hline Non-viral & 1 & & & & & \\
\hline Viral & $1.731(0.801-3.740)$ & 0.163 & & & & \\
\hline \multicolumn{7}{|c|}{ Non-tumor pathology } \\
\hline Cirrhosis & 1 & & & & & \\
\hline Others & $0.904(0.603-1.356)$ & 0.626 & & & & \\
\hline \multicolumn{7}{|c|}{ PNCK IHC expression } \\
\hline Low & 1 & & 1 & & & \\
\hline High & $1.554(1.000-2.413)$ & 0.050 & $1.214(0.753-1.956)$ & 0.426 & & \\
\hline$P N C K$ mRNA & & & & & & \\
\hline Low & 1 & & & & 1 & \\
\hline High & $2.695(1.712-4.244)$ & $<0.001$ & & & $1.908(1.183-3.078)$ & 0.008 \\
\hline
\end{tabular}

HR, Hazard Ratio; CI, Confidence Interval; AJCC, American Joint Committee on Cancer; BCLC, Barcelona Clinic Liver Cancer; AFP, $\alpha$-fetoprotein; PNCK, pregnancy up-regulated non-ubiquitous calmodulin kinase; IHC, immunohistochemistry. 
Table III. Univariate and multivariate analysis for recurrence-free survival.

\begin{tabular}{|c|c|c|c|c|c|c|}
\hline \multirow[t]{3}{*}{ Category } & \multicolumn{6}{|c|}{ Recurrence free survival } \\
\hline & \multicolumn{2}{|c|}{ Univariate } & \multicolumn{2}{|c|}{ Multivariate } & \multicolumn{2}{|c|}{ Multivariate } \\
\hline & HR $(95 \%$ CI $)$ & $p$-Value & $\mathrm{HR}(95 \% \mathrm{CI})$ & $p$-Value & $\operatorname{HR}(95 \% \mathrm{CI})$ & $p$-Value \\
\hline \multicolumn{7}{|l|}{ Age $(y)^{*}$} \\
\hline$\leq 55$ & 1 & & & & & \\
\hline$>55$ & $1.045(0.769-1.420)$ & 0.780 & & & & \\
\hline \multicolumn{7}{|l|}{ Gender } \\
\hline Female & 1 & & & & & \\
\hline Male & $0.971(0.667-1.412)$ & 0.877 & & & & \\
\hline \multicolumn{7}{|c|}{ Tumor size $(\mathrm{cm})$} \\
\hline$\leq 5$ & 1 & & 1 & & 1 & \\
\hline$>5$ & $1.824(1.341-2.479)$ & $<0.001$ & $0.997(0.688-1.444)$ & 0.988 & $0.846(0.573-1.248)$ & 0.398 \\
\hline \multicolumn{7}{|c|}{ Microvascular invasion } \\
\hline Absent & 1 & & 1 & & 1 & \\
\hline Present & $2.341(1.714-3.196)$ & $<0.001$ & $1.178(0.798-1.740)$ & 0.410 & $1.349(0.891-2.040)$ & 0.157 \\
\hline \multicolumn{7}{|c|}{ Major portal vein invasion } \\
\hline Absent & 1 & & 1 & & 1 & \\
\hline Present & $4.242(2.345-7.675)$ & $<0.001$ & $1.030(0.541-1.960)$ & 0.929 & $0.779(0.351-1.731)$ & 0.779 \\
\hline \multicolumn{7}{|c|}{ Intrahepatic metastasis } \\
\hline Absent & 1 & & 1 & & 1 & \\
\hline Present & $4.323(3.115-6.000)$ & $<0.001$ & $5.113(3.647-7.169)$ & $<0.001$ & $5.185(3.532-7.611)$ & $<0.001$ \\
\hline \multicolumn{7}{|l|}{ Multicentricity } \\
\hline Absent & 1 & & & & & \\
\hline Present & $0.640(0.338-1.212)$ & 0.171 & & & & \\
\hline \multicolumn{7}{|c|}{ Edmonson grade } \\
\hline I-II & 1 & & 1 & & 1 & \\
\hline III & $2.277(1.395-3.716)$ & 0.001 & $1.507(0.916-2.480)$ & 0.106 & $1.414(0.797-2.507)$ & 0.237 \\
\hline \multicolumn{7}{|l|}{ AJCC T stage } \\
\hline $\mathrm{T} 1$ & 1 & & & & & \\
\hline $\mathrm{T} 2$ to $\mathrm{T} 4$ & $1.656(1.083-2.531)$ & 0.020 & & & & \\
\hline \multicolumn{7}{|l|}{ BCLC stage } \\
\hline 0 to $\mathrm{A}$ & 1 & & & & & \\
\hline B to $\mathrm{C}$ & $1.697(1.259-2.287)$ & 0.001 & & & & \\
\hline \multicolumn{7}{|c|}{ Serum albumin level } \\
\hline$>3.5 \mathrm{~g} / \mathrm{dl}$ & 1 & & & & & \\
\hline$\leq 3.5 \mathrm{~g} / \mathrm{dl}$ & $1.004(0.652-1.546)$ & 0.985 & & & & \\
\hline \multicolumn{7}{|c|}{ Serum AFP level } \\
\hline$\leq 200 \mathrm{ng} / \mathrm{ml}$ & 1 & & 1 & & 1 & \\
\hline$>200 \mathrm{ng} / \mathrm{ml}$ & $1.779(1.314-2.410)$ & $<0.001$ & $1.294(0.944-1.775)$ & 0.109 & $1.150(0.795-1.665)$ & 0.458 \\
\hline \multicolumn{7}{|l|}{ Etiology } \\
\hline Non-viral & 1 & & 1 & & 1 & \\
\hline Viral & $1.997(1.134-3.519)$ & 0.017 & $1.583(0.871-2.877)$ & 0.132 & $1.864(0.938-3.703)$ & 0.076 \\
\hline \multicolumn{7}{|c|}{ Non-tumor pathology } \\
\hline Cirrhosis & 1 & & & & & \\
\hline Others & $0.787(0.583-1.061)$ & 0.116 & & & & \\
\hline \multicolumn{7}{|c|}{ PNCK IHC expression } \\
\hline Low & 1 & & 1 & & & \\
\hline High & $1.631(1.189-2.237)$ & 0.002 & $1.213(0.866-1.697)$ & 0.261 & & \\
\hline$P N C K$ mRNA & & & & & & \\
\hline Low & 1 & & & & 1 & \\
\hline High & $1.747(1.245-2.452)$ & 0.001 & & & $1.078(0.711-1.634)$ & 0.722 \\
\hline
\end{tabular}

HR, Hazard Ratio; CI, Confidence Interval; AJCC, American Joint Committee on Cancer; BCLC, Barcelona Clinic Liver Cancer; AFP, $\alpha$-fetoprotein; PNCK, pregnancy up-regulated non-ubiquitous calmodulin kinase; IHC, immunohistochemistry. 
PNCK at both the protein and mRNA level was associated with aggressive clinicopathological parameters such as high Edmondson grade, presence of microvascular invasion, presence of intrahepatic metastasis and high AFP level, as well as shorter RFS and DSS, in a large cohort of HCC patients with long-term follow-up.

$P N C K$ is a novel member of calmodulin kinase, which is involved in diverse biological processes including cell-cycle control, transcriptional regulation, neurotransmitter release and muscle contraction (7). PNCK is located in Xq28 and its protein expression differs with regards to organs, and is highest in brain, and moderate to low in hormone-related tissues such as uterus, ovary, testis and mammary gland, or other tissues such as stomach, heart and skeletal muscle (7). There have been a few previous studies regarding $P N C K$ expression in cancer. $P N C K$ overexpression was found in more than $30 \%$ of HER-2 amplified breast cancer, and induced proliferation, cell-cycle progression and clonogenicity as well as trastuzumab resistance through phosphatase and tensin homologue (PTEN)-mediated process (8). In clear cell renal cell carcinoma, $P N C K$ was one of the mostly overexpressed genes by massive parallel sequencing analysis (18), and PNCK expression at the protein and mRNA levels were higher in tumor than non-tumor samples, and associated with higher Fuhrman grade, larger tumor size and advanced $\mathrm{T}$ and $\mathrm{N}$ stage, as well as a poor prognosis (10). In a recent study in nasopharyngeal carcinoma, PNCK expression at the protein and mRNA levels was found increased in tumor tissues, and knockdown of $P C N K$ inhibited proliferation and induced apoptosis by regulating $P I 3 K / A K T / m T O R$ signaling pathway by in vitro and in vivo experiments (9) .

In this study, we are the first to report the overexpression of PNCK and its association with poor prognosis in HCC, and these results are highly consistent with results from previous studies in other cancers. Our study provides clinical evidence suggesting PNCK as a potential therapeutic tool targeting HCC. Previous studies revealed that PNCK mediates degradation of epidermal growth factor receptor (EGFR) protein, which might be a promising target for EGFR-regulated oncogenesis $(19,20)$. EGFR overexpression was found in up to $68 \%$ of the HCC cohort, and was related with aggressiveness of tumor $(21,22)$. It was found mainly in poorly differentiated HCC, and was correlated with high Ki-67 proliferative index, advanced stage, presence of intrahepatic metastasis and poorer prognosis $(21,22)$. However, monotherapy using cetuximab, the monoclonal antibody against EGFR, showed disappointing results to date in HCC patients (23). A case report of complete remission of unresectable HCCs treated with another anti-EGFR antibody nimotuzumab have been reported, suggesting $E G F R$ as a potential therapeutic target in HCC (23). The implication for PNCK expression for prediction for targeted therapy, including anti-EGFR drugs, needs further examination.
In conclusion, we demonstrated up-regulation of PNCK expression in HCCs and its association with poor prognosis. PNCK might be a prognostic biomarker of HCCs, and could be a potential candidate therapeutic target.

\section{Conflicts of Interest}

None of the Authors have any conflicts of interest to declare regarding this study.

\section{Authors' Contributions}

Conception and design: SYH; Acquisition of data: YAC, SC, SP, CP, SYH; Analysis and interpretation of data: YAC, SYH; Drafting the article: YAC, SYH; Revising and final approval of the article to be published: YAC, SC, SP, CP, SYH; All Authors read and approved the final manuscript.

\section{Acknowledgements}

This study was funded by Samsung Medical Center intramural grant (\#SMO1161731) and the Basic Science Research Program through the National Research Foundation of Korea (NRF), funded by the Ministry of Education (NRF-2017R1C1B5017890).

\section{References}

1 Bruix J, Qin S, Merle P, Granito A, Huang Y-H, Bodoky G, Pracht M, Yokosuka O, Rosmorduc O, Breder V, Gerolami R, Masi G, Ross PJ, Song T, Bronowicki J-P, Ollivier-Hourmand I, Kudo M, Cheng A-L, Llovet JM, Finn RS, LeBerre M-A, Baumhauer A, Meinhardt G and Han G: Regorafenib for patients with hepatocellular carcinoma who progressed on sorafenib treatment (RESORCE): a randomised, double-blind, placebocontrolled, phase 3 trial. The Lancet 389(10064): 56-66, 2017. PMID: 27932229. DOI: 10.1016/s0140-6736(16)32453-9

2 Kudo M, Finn RS, Qin S, Han K-H, Ikeda K, Piscaglia F, Baron A, Park J-W, Han G, Jassem J, Blanc JF, Vogel A, Komov D, Evans TRJ, Lopez C, Dutcus C, Guo M, Saito K, Kraljevic S, Tamai T, Ren M and Cheng A-L: Lenvatinib versus sorafenib in first-line treatment of patients with unresectable hepatocellular carcinoma: a randomised phase 3 non-inferiority trial. The Lancet 391(10126): 1163-1173, 2018. PMID: 29433850. DOI: 10.1016/s0140-6736(18)30207-1

3 Zhu AX, Finn RS, Edeline J, Cattan S, Ogasawara S, Palmer D, Verslype C, Zagonel V, Fartoux L, Vogel A, Sarker D, Verset G, Chan SL, Knox J, Daniele B, Webber AL, Ebbinghaus SW, Ma J, Siegel AB, Cheng A-L, Kudo M, Alistar A, Asselah J, Blanc J-F, Borbath I, Cannon T, Chung K, Cohn A, Cosgrove DP, Damjanov N, Gupta M, Karino Y, Karwal M, Kaubisch A, Kelley R, Van Laethem J-L, Larson T, Lee J, Li D, Manhas A, Manji GA, Numata K, Parsons B, Paulson AS, Pinto C, Ramirez R, Ratnam S, Rizell M, Rosmorduc O, Sada Y, Sasaki Y, Stal PI, Strasser S, Trojan J, Vaccaro G, Van Vlierberghe H, Weiss A, Weiss K-H and Yamashita T: Pembrolizumab in patients with advanced hepatocellular carcinoma previously treated with sorafenib (KEYNOTE-224): a non-randomised, open-label phase 2 trial. The Lancet Oncology 19(7): 940-952, 2018. PMID: 29875066. DOI: 10.1016/s1470-2045(18)30351-6 
4 El-Khoueiry AB, Sangro B, Yau T, Crocenzi TS, Kudo M, Hsu C, Kim T-Y, Choo S-P, Trojan J, Welling TH, Meyer T, Kang YK, Yeo W, Chopra A, Anderson J, dela Cruz C, Lang L, Neely J, Tang H, Dastani HB and Melero I: Nivolumab in patients with advanced hepatocellular carcinoma (CheckMate 040): an openlabel, non-comparative, phase $1 / 2$ dose escalation and expansion trial. The Lancet 389(10088): 2492-2502, 2017. PMID: 28434648. DOI: 10.1016/s0140-6736(17)31046-2

5 Qin LX and Tang ZY: Recent progress in predictive biomarkers for metastatic recurrence of human hepatocellular carcinoma: a review of the literature. J Cancer Res Clin Oncol 130(9): 497513, 2004. PMID: 15205947. DOI: 10.1007/s00432-004-0572-9

6 Kitagawa A, Masuda T, Takahashi J, Tobo T, Noda M, Kuroda Y, Hu Q, Kouyama Y, Kobayashi Y, Kuramitsu S, Sato K, Fujii A, Yoshikawa Y, Wakiyama H, Shimizu D, Tsuruda Y, Eguchi H, Doki Y, Mori M and Mimori K: KIF15 Expression in tumorassociated monocytes is a prognostic biomarker in hepatocellular carcinoma. Cancer Genomics Proteomics 17(2): 141-149, 2020. PMID: 32108036. DOI: 10.21873/cgp.20174

7 Gardner HP, Rajan JV, Ha SI, Copeland NG, Gilbert DJ, Jenkins NA, Marquis ST and Chodosh LA: Cloning, characterization, and chromosomal localization of Pnck, a $\mathrm{Ca}(2+) /$ calmodulindependent protein kinase. Genomics 63(2): 279-288, 2000. PMID: 10673339. DOI: 10.1006/geno.1999.6091

8 Deb TB, Zuo AH, Barndt RJ, Sengupta S, Jankovic R and Johnson MD: Pnck overexpression in HER-2 gene-amplified breast cancer causes Trastuzumab resistance through a paradoxical PTEN-mediated process. Breast Cancer Res Treat 150(2): 347-361, 2015. PMID: 25773930. DOI: 10.1007/s10549$015-3337-z$

9 Xu Y, Wang J, Cai S, Chen G, Xiao N, Fu Y, Chen Q and Qiu $\mathrm{S}$ : PNCK depletion inhibits proliferation and induces apoptosis of human nasopharyngeal carcinoma cells in vitro and in vivo. J Cancer 10(27): 6925-6932, 2019. PMID: 31839828. DOI: 10.7150/jca.33698

10 Wu S, Lv Z, Wang Y, Sun L, Jiang Z, Xu C, Zhao J, Sun X, Li X, Hu L, Tang A, Gui Y, Zhou F, Cai Z and Wang R: Increased expression of pregnancy up-regulated non-ubiquitous calmodulin kinase is associated with poor prognosis in clear cell renal cell carcinoma. PLoS One 8(4): e59936, 2013. PMID: 23634203. DOI: 10.1371/journal.pone.0059936

11 Amin MB ES, Greene F, Byrd DR, Brookland RK, Washington MK, et al: AJCC Cancer Staging Manual. 8th ed. New York, Springer, 2017.

12 Llovet JM, Brú C and Bruix J: Prognosis of hepatocellular carcinoma: the BCLC staging classification. Semin Liver Dis 19(3): 329-338, 1999. PMID: 10518312. DOI: 10.1055/s-20071007122

13 Kumada T, Nakano S, Takeda I, Sugiyama K, Osada T, Kiriyama S, Sone Y, Toyoda H, Shimada S, Takahashi M and Sassa T: Patterns of recurrence after initial treatment in patients with small hepatocellular carcinoma. Hepatology 25(1): 87-92, 1997. PMID: 8985270. DOI: 10.1053/jhep.1997.v25.pm0008985270

14 Ha SY, Kim JH, Yang JW, Kim J, Kim B and Park CK: The Overexpression of CCAR1 in Hepatocellular Carcinoma Associates with Poor Prognosis. Cancer Res Treat 48(3): 10651073, 2016. PMID: 26511806. DOI: 10.4143/crt.2015.302
15 Lee T, Park CK and Ha SY: Prognostic role of apelin receptor expression in hepatocellular carcinoma treated with curative surgical resection. Anticancer Res 39(6): 3025-3031, 2019. PMID: 31177144. DOI: 10.21873/anticanres.13435

16 Lim HY, Sohn I, Deng S, Lee J, Jung SH, Mao M, Xu J, Wang K, Shi S, Joh JW, Choi YL and Park CK: Prediction of diseasefree survival in hepatocellular carcinoma by gene expression profiling. Ann Surg Oncol 20(12): 3747-3753, 2013. PMID: 23800896. DOI: 10.1245/s 10434-013-3070-y

17 Camp RL, Dolled-Filhart M and Rimm DL: X-Tile. A New bioinformatics tool for biomarker assessment and outcome-based cut-point optimization. Clin Cancer Res 10(21): 7252-7259, 2004. PMID: 15534099. DOI: 10.1158/1078-0432.Ccr-04-0713

18 Zhou L, Chen J, Li Z, Li X, Hu X, Huang Y, Zhao X, Liang C, Wang Y, Sun L, Shi M, Xu X, Shen F, Chen M, Han Z, Peng Z, Zhai Q, Chen J, Zhang Z, Yang R, Ye J, Guan Z, Yang H, Gui Y, Wang J, Cai Z and Zhang X: Integrated profiling of microRNAs and mRNAs: microRNAs located on Xq27.3 associate with clear cell renal cell carcinoma. PLoS One 5(12): e15224, 2010. PMID: 21253009. DOI: 10.1371/journal.pone.0015224

19 Deb TB, Coticchia CM, Barndt R, Zuo H, Dickson RB and Johnson MD: Pregnancy-upregulated nonubiquitous calmodulin kinase induces ligand-independent EGFR degradation. Am J Physiol Cell Physiol 295(2): C365-C377, 2008. PMID: 18562482. DOI: 10.1152/ajpcell.00449.2007

20 Deb TB, Zuo AH, Wang Y, Barndt RJ, Cheema AK, Sengupta S, Coticchia CM and Johnson MD: Pnck induces ligand-independent EGFR degradation by probable perturbation of the Hsp90 chaperone complex. Am J Physiol Cell Physiol 300(5): C11391154, 2011. PMID: 21325639. DOI: 10.1152/ajpcell.00167.2010

21 Kira S, Nakanishi T, Suemori S, Kitamoto M, Watanabe Y and Kajiyama G: Expression of transforming growth factor alpha and epidermal growth factor receptor in human hepatocellular carcinoma. Liver 17(4): 177-182, 1997. PMID: 9298487. DOI: 10.1111/j.1600-0676.1997.tb00803.x

22 Ito Y, Takeda T, Sakon M, Tsujimoto M, Higashiyama S, Noda $\mathrm{K}$, Miyoshi E, Monden M and Matsuura N: Expression and clinical significance of erb-B receptor family in hepatocellular carcinoma. Br J Cancer 84(10): 1377-1383, 2001. PMID: 11355950. DOI: $10.1054 /$ bjoc. 2000.1580

23 Song P, Yang J, Li X, Huang H, Guo X, Zhou G, Xu X, Cai Y, Zhu M, Wang P, Zhao S and Zhang D: Hepatocellular carcinoma treated with anti-epidermal growth factor receptor antibody nimotuzumab: A case report. Medicine (Baltimore) 96(39): e8122, 2017. PMID: 28953642. DOI: 10.1097/md.0000000000008122

Received August 25, 2020

Revised September 25, 2020

Accepted October 5, 2020 